\title{
Optimization Approach To Optimal Power Efficient Based On Cluster Top Option In Wireless Sensor Networks
}

\author{
${ }^{1}$ Hasanien Ali Talib, ${ }^{2}$ Dr.Raya Alothman, ${ }^{3}$ Marwah Khaleel Farhan \\ ${ }^{1}$ Computer Science Department, College of Pure Sciences for Education,University of Mosul, Mosul, Iraq \\ hasanien.ali@uomosul.edu.iq \\ ${ }^{2}$ Computer Science Department, College of Pure Sciences for Education, University of Mosul, Mosul, Iraq \\ dr.raya.alothman@uomosul.edu.iq \\ ${ }^{3}$ Scholarships and Cultural Relations Directorate Ministry of Higher Education and Scientific Research, Baghdad, \\ Iraq. \\ marwah.k.farhan@gmail.com
}

Article History: Received:11 January 2021; Accepted: 27 February 2021; Published online: 5 April 2021

\begin{abstract}
Wireless sensor network is the significant element in pervasive computing for various distributed applications which divides the resources or data among the peers between the sensor nodes to managing the bandwidth of network, node participants of the network, processing powers of the nodes. During the process of the distribution of the data, the one to one environment of the sensor networks, computation complexity and decentralization is the important issues because it completely reduces the entire system performance. So, this paper implementing the system to distributing the datum among the nodes in one to one environment using the distributed data clustered through the help about the honey-bee optimization with genetic algorithm approach. The analyzes of approach of cluster and relationship of the neighboring managing on the nodes of peer of the sensors in order to assist to decentralization process on the dynamic environment. Further a clustering search space is identified and each node is evaluated with the help of position, velocity, distance values which is examined in terms of using fitness value. Then a performance at a system is analyzed using the results of experimental while discussions such as packet delivery ratio, energy consumption and total number of alive nodes.
\end{abstract}

Keywords: Genetic algorithm, Peer to peer nodes, packet delivery ratio, cluster head, Wireless sensor networks.

\section{INTRODUCTION}

Wireless Sensor Networks (WSN) was considered as an effective communication process because it comprises of groups of sensor nodes that monitor the condition of the environment, sound, pressure and temperature continuously [1]. The collected information is transmitted from sender to receiver with the help of the sensor activities. Due to the effective communication process of WSN, it has been utilized by several applications such as health monitoring, control mechanisms, surveillance and industrial monitoring process [2]. During the communication process, the sensor network incorporated with several characteristics such as ability to withstand in difficult environmental situations, homogeneity of nodes, mobility of nodes, heterogeneity of nodes, power consumption also the network must have the effective cross layer designs [3]. By following the above described characteristics of WSN while starting the information transmission, the networks has several advantages such as network flexibility, helpful in society related applications, minimize the information transmission cost [4].

Even though the network utilizes those characteristics effective manner with different environment, sensor network must handle various issues such as intermediate attacks, low communication speed, distraction by other device and battery or energy consumption at the time of information transmitting from source to destination nodes [5]. Among the various disadvantages, energy consumption is one of the major issues because higher energy utilization leads to reduce the network lifetime as a result to nodes failure, link failure, and network failure. So, different researchers analyze routing methodologies for managing the energy issues in WSN. [6] Has developed the ant colony-based Meta heuristic protocol of power efficient routing to increase the network-lifetime also helps to detect the shortest path from source to destination node. during the process, ARNet aids to balance the nodes consume of power of the. 
The author introduced system successfully manages the network lifetime and the system Excellency was evaluated aided by the results of simulation which is compared with the traditional routing algorithms. In [7], the author proposed an algorithm for the cluster selection of heads by mixing the genetic algorithm and fuzzy logic. This approach uses the fuzzy logic for choosing the better cluster heads to reach the base station by considering the input factors such as energy, centrality and density. Cluster heads' number and place were found out using the Chaotic based GA[8]. Even though these routing methods detect the shortest path by consuming the effective energy, but these methods are sometimes fail to meet network life time due to large scale of network. So, in these uses the different energy efficient routing protocols such as Particle with Ant Swarm Optimization (PASO) technique, CoEvolutionary Particle Swarm Optimization (CPSO) algorithm and Honey Bee Optimization With Genetic Algorithm for improving network lifetime[9]. The introduce energy efficiency method excellence is evaluated with the help of implementation process which reduces minimum network life time also improves the packet delivery ratio. Other solution to overcome the loss energy is using appropriate caching techniques to reduce transmission thereby reduce energy consumption and the time delay when fetching the requested data by using an enhanced grouping technique inside each cluster [10].

\subsection{PROBLEM DEFINITION}

Wireless Sensor Networks (WSN) has group of of sensor nodes that helps in data transmitting from source to destination node. During the data transmission process, it has several characteristics such as power consumption, energy consumption, heterogeneous node, scalability and so on [11]. Even though the WSN consists of various features, the energy and network lifetime is one of the major issues because it causes the serious problems such as node failure, link failure, data failure and false data transmission [12]. Hence the major aim of the study is to eliminate the high utilization of the energy as well as managing high network lifetime thru th utilization of various optimized clustering and routing methodologies. Clustering method [13] analyzes every node present in the network before data transmitting. The formed cluster eliminates the minimum energy consumed node also it improves the transmitted data ratio with manner.

\subsection{PROPOSED WORK}

There are many methods to efficient energy and load balancing in wireless sensor network such as short and active path choice as well as caching technique which consider efficient solution to decreasing energy consumption because it reduces the transmission of redundant packets. The nodes transmit just required packet thereby reduce the packets transmission and increasing lifetime of battery inside nodes which improves efficient of whole network [10]. Caching technique always use cooperative caching, which mean multiple sensor nodes share and coordinate cache data to cut communication cost(energy consumption) and exploit the aggregate cache space of cooperating sensors .

If caching Implemented optimally can reduce a lot of network traffic and helps in providing higher data availability to the sink without need more transmission that mean no more consumption for network resources. in next table we compare between Distributed TCP Caching (DTC) protocol which is used in caching techniques to reduce the energy consumption[20].

The another way by using load balancing protocols like LEACH protocol which has been designed to reduce power consumption and make it more efficient thereby enhance the wireless sensor network lifetime and energy of sensor nodes [19].

In this research work analyze the effective clustering methods for eliminating the higher energy utilization as well as managing the lifetime of the network [14] within WSN. This objective is achieved by applying three different clustering-based routing methodologies which are listed as follows,

- Optimal Energy Efficient Cluster Head Selection (OEECHS) for WSN Particle with Ant Swarm Optimization (PASO).

- Co-Evolutionary Particle Swarm Optimization with Fuzzy Multiple Parameter Decision-Making for Avoid Load And Bandwidth Consumption in WSN.

- HoneyBee Optimization with Genetic Algorithm for Avoid Load And Bandwidth Consumption in WSN 


\begin{tabular}{|c|c|c|c|c|c|c|}
\hline Technique & classification & $\begin{array}{c}\text { Power } \\
\text { usage }\end{array}$ & $\begin{array}{c}\text { Data } \\
\text { aggregation }\end{array}$ & $\begin{array}{c}\text { Query } \\
\text { based }\end{array}$ & $\begin{array}{c}\text { Delay } \\
\text { Time }\end{array}$ & Scalability \\
\hline $\begin{array}{c}\text { Load } \\
\text { balancing } \\
\text { LEACH } \\
\text { protocol }\end{array}$ & $\begin{array}{c}\text { Hierarchical(all } \\
\text { sensors send data } \\
\text { to sink node }\end{array}$ & high & Yes & No & good & Good \\
\hline $\begin{array}{c}\text { Cache } \\
\text { technique } \\
\text { DTC }\end{array}$ & $\begin{array}{c}\text { Hierarchical(all } \\
\text { sensors send data } \\
\text { to sink node }\end{array}$ & $\begin{array}{c}\text { Less than } \\
\text { LEACH }\end{array}$ & Yes & Yes & good & limited \\
\hline $\begin{array}{c}\text { Optimal } \\
\text { Energy } \\
\text { Efficient } \\
\text { Cluster } \\
\text { Head } \\
\text { Selection }\end{array}$ & $\begin{array}{c}\text { Hierarchical(all } \\
\text { sensors send data } \\
\text { to cluster head } \\
\text { node }\end{array}$ & $\begin{array}{c}\text { Best than } \\
\text { other }\end{array}$ & Yes & Yes & $\begin{array}{c}\text { Best } \\
\text { because it } \\
\text { select short } \\
\text { path }\end{array}$ & Good \\
\hline
\end{tabular}

Table ( ) comparison between different techniques which enhance energy of nodes in WSN

\section{CONTRIBUTION OF THE RESEARCH}

In this research work, network lifetime and energy has been managed by selecting optimized cluster which is done by using the Particle with Ant Swarm Optimization (PASO) technique, Co-Evolutionary Particle Swarm Optimization (CPSO) algorithm and Honeybee Optimization with Genetic Algorithm (HBPGA). Each and every optimization algorithm, has specific fitness function while selecting the cluster head as well as cluster member tht used to manages the network energy with effective manner which is explained as follows.

\subsection{Optimal Energy Efficient Cluster Head Selection (OEECHS) for Wireless Sensor Network}

In this research work, particle with ant swarm optimization-based clustering head selection process is created for managing the energy during data transmitting from source to destination nodes. Before making the cluster head selection process, few assumptions are considered in the research work such as, sink node position has been located according to the centre coordinate value within the sensing area, selected clusters are represented in the defined cluster region along with cluster radius and so on[15-16]. After making the proper assumptions, search space is defined in the network area for selecting the optimal cluster head depending on the fitness value of the particle. During this process, each node or particle position and velocity value is estimated as follows,

$\mathbf{x}_{\mathbf{i}}=\left(\mathbf{x}_{\mathrm{i} 1}, \mathbf{x}_{\mathrm{i} 2}, \ldots \mathbf{x}_{\mathrm{iM}}\right)^{\mathbf{T}}$

$\mathbf{v}_{\mathbf{i}}=\left(\mathbf{v}_{\mathrm{i} 1}, \mathbf{v}_{\mathrm{i} 2}, \ldots . \mathbf{v}_{\mathrm{iM}}\right)^{\mathbf{T}}$

Where $\mathrm{i}=1,2, \ldots \ldots, \mathrm{N}$ and $\mathrm{M}$ indicates the dimension. Besides $\mathrm{x}_{\mathrm{ij}}(\mathrm{t})$ represents the ith particle position and $\mathrm{v}_{\mathrm{ij}}(\mathrm{t})$ represents the velocity in jth dimension at time $\mathrm{t}[21]$.

The computed particle position value is continuously changed because, one particle is communicated with the neighboring particles which helps to improve the overall cluster head selection process. The neighboring node communication process is initiated via ant searching process it utilizes the wandering process also increase the count value depending on the searching. Moreover, the PSO algorithm has been processed until the maximum iteration Tmax so as to explore the best candidates as the locations of cluster head. So, it utilizes the below equations towards updating the particles' velocity and position[22].

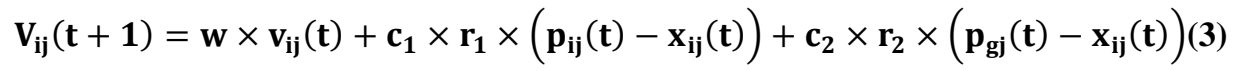

$\mathbf{x}_{\mathrm{ij}}(\mathbf{t}+\mathbf{1})=\mathbf{x}_{\mathrm{ij}}(\mathrm{t})+\mathbf{v}_{\mathrm{ij}}(\mathbf{t}+\mathbf{1})$ 
where $\mathrm{c} 1$ and $\mathrm{c} 2$ represents the acceleration constants, $\mathrm{w}$ is the interia weight and $\mathrm{r} 1$ and $\mathrm{r} 2$ represents the random variables produced randomly between $[0,1]$. The interia weight minimize the search space of the particle adaptively using

$\mathbf{w}_{\mathbf{t}}=\left(\mathbf{w}_{\text {max }}-\mathbf{w}_{\text {min }}\right) \times \frac{\text { maxiteration }-\mathbf{t}}{\text { maxiteration }}+\mathbf{w}_{\text {min }}$

The pij represents the local best position of the particle I at the jth dimension and the pgj represents the global best position respect to all particles in the cluster at the jth dimension[23]. From the updated pheromone value and particle initialization process, gbest position of the particle is estimated for each iteration using mean, standard deviation value which is estimated as follows,

$$
a_{i}(t)=N\left(p_{g}(t), \sigma\right)
$$

In eqn (6), the algorithm produce the solution vector $a_{i}(t)$, which fulfil the Guassian distributions with $p_{g}(t)$ and $\sigma$, which is to begin with at $\mathrm{t}=1$ value of $\sigma=1$ and at end of every generation it will be updated as $\sigma=\sigma \times d$, where $\mathrm{d}$ is a factor to generate a better position within this boundary. The fitness function value $a_{i}(t)$ has been measured using the equation (11) by changing the position of $p_{i}(t)$ (Current position of particle i). If $\mathrm{F}\left(a_{i}(t)\right)<\mathrm{F}\left(x_{i}(t)\right.$ ) then $x_{i}(t)=a_{i}(t)$ and $F\left(x_{i}(t)\right)=F\left(a_{i}(t)\right)$. The ant colony based pheromone guided search has been incorporated with the PSO to act locally so as to synchronize the position of the particles in space to acquire the optimal domain of the fitness function which is estimated as follow,

$\mathbf{F}\left(\mathbf{x}_{\mathrm{i}}(\mathrm{t})\right)=$ optimize $\left(\alpha_{1} \mathbf{X}_{1}+\alpha_{2} \mathbf{X}_{2}+\alpha_{3} \mathbf{X}_{3}+\left(1-\alpha_{1}-\alpha_{2}-\alpha_{3}\right) X_{4}\right)$

Subject to:

$\mathbf{X}_{1}=\frac{\left(\left(\sum_{\mathrm{j}=1}^{\mathrm{k}} \mathbf{d}_{\mathrm{j}, \mathrm{i}}\right)+\mathrm{d}_{\mathrm{i}, \mathrm{s}}\right)}{\mathrm{k}+\mathbf{1}}$

$\mathbf{X}_{2}=\sum_{\mathbf{i}}^{\mathrm{N}} \mathbf{E}\left(\mathbf{p}_{\mathbf{i}}\right) / \sum_{\mathbf{j}=1}^{\mathrm{k}} \mathbf{E}\left(\mathbf{n}_{\mathbf{j}}\right), \mathbf{E}_{\min } \leq \mathbf{E}\left(\mathbf{n}_{\mathbf{j}}\right) \leq \mathbf{E}_{\max }$

$\mathbf{X}_{3}=\mathbf{N}_{\mathrm{deg}}\left(\mathbf{p}_{\mathrm{i}}\right), \mathbf{0}<\boldsymbol{\alpha}_{1}, \boldsymbol{\alpha}_{2}, \boldsymbol{\alpha}_{3}<\mathbf{1}$

$X_{4}=1 / H\left(p_{i}\right), H\left(p_{i}\right) \geq 1$, and $\alpha_{1} \leq \alpha_{2} \leq \alpha_{3}$

Where $\alpha_{1}, \alpha_{2}, \alpha_{3}$ are the weighting parameters of the respective factor[24].

According to the above discussions, the fitness value, energy level of each node, distance between nodes are used to choose optimized cluster head. From the above equation it has been clear that the X1 is the average distance between pi out of the other member nodes in cluster and the sink node. X2 is the computation of particle power corresponding to other nodes. X3 is the node degree associated with the particle i. X4 is the conjugate of the head count of particle i. By the increase of head count, selection probability for selecting that particular node will get decreases. Moreover, after the completion of every round, the particle optimizes the function of fitness with the help of pheromone guided search has been selected as the most suitable position of the cluster head node. The selected cluster head via data transmission improves by utilizing minimum energy. Even though the method successfully detects the cluster head in network, it has been further improved by using the algorithm of Co-Evolutionary Particle Swarm Optimization (CEPSO) that is explained in following section[25].

\subsection{Co-Evolutionary Particle Swarm Optimization with Fuzzy Multiple Parameter Decision-Making for Avoid Load and Bandwidth Consumption in WSN}

This work utilizes the fuzzy multiple parameter decision making while analyzing the co-evolutionary particle swarm optimization clustering head selection. At the time cluster selection, computed threshold value is compared with different parameters such as Average Energy of the Network, Initial Energy, Rate of Power Consumption and residual energy [17-18]. First the searching space is identified, mutant strategy and mixed inertia weight value of each node is estimated as follows, that helps to avoid the load and bandwidth of the network.

$$
\boldsymbol{w}=\boldsymbol{w}_{\text {max }}-\left(\boldsymbol{w}_{\max }-\boldsymbol{w}_{\text {min }}\right) * \frac{\text { iter }}{\text { maxiter }}
$$


The estimated mutant strategy value is analyzed up to maximum $10 \%$ iteration, if the computed value is remains unchanged than half of the nodes are again re-initiated as normal nodes. Then cluster head and normal node distance was calculated which must be normally equal. When a nominated cluster head fulfills both the conditions stated above an even cluster is designed is optimal function is defined as:

$$
F=\min \left[\operatorname{mean}\left(\sum_{i=1}^{m} D_{j i}-j_{c h}\right) * \operatorname{mean}\left(\operatorname{var}\left(\sum_{i=1}^{m} D_{j i}-j_{c h}\right)\right)\right]
$$

In Cluster selection approaches are accessible to avoid impulsive immobility whereas stagnation at a late stage is not protected. The value of cluster constants $c_{1}$ and $c_{2}$ utilized by

$$
c_{1}=c_{2}=c_{\text {initial }}+\operatorname{random}(0,1)
$$

Where $c_{\text {initial }}$ is 2 and the value of $c_{1} \& c_{2}$ deceptions between $2-3$.The value of inertia can be computed by using following eqn (15).

$$
w=w_{\text {initial }}+\frac{\text { random }(0,50)}{100}
$$

Where $w_{\text {initial }}$ is initially defined as 0.4 . Hereafter, consider the inertia value ranges is between $0.4-0.9$. The computed values are fed into the fuzzy multiparameter decision making process for overcoming the bandwidth and load issue. Then the decision making process structure is shown in figure 1.

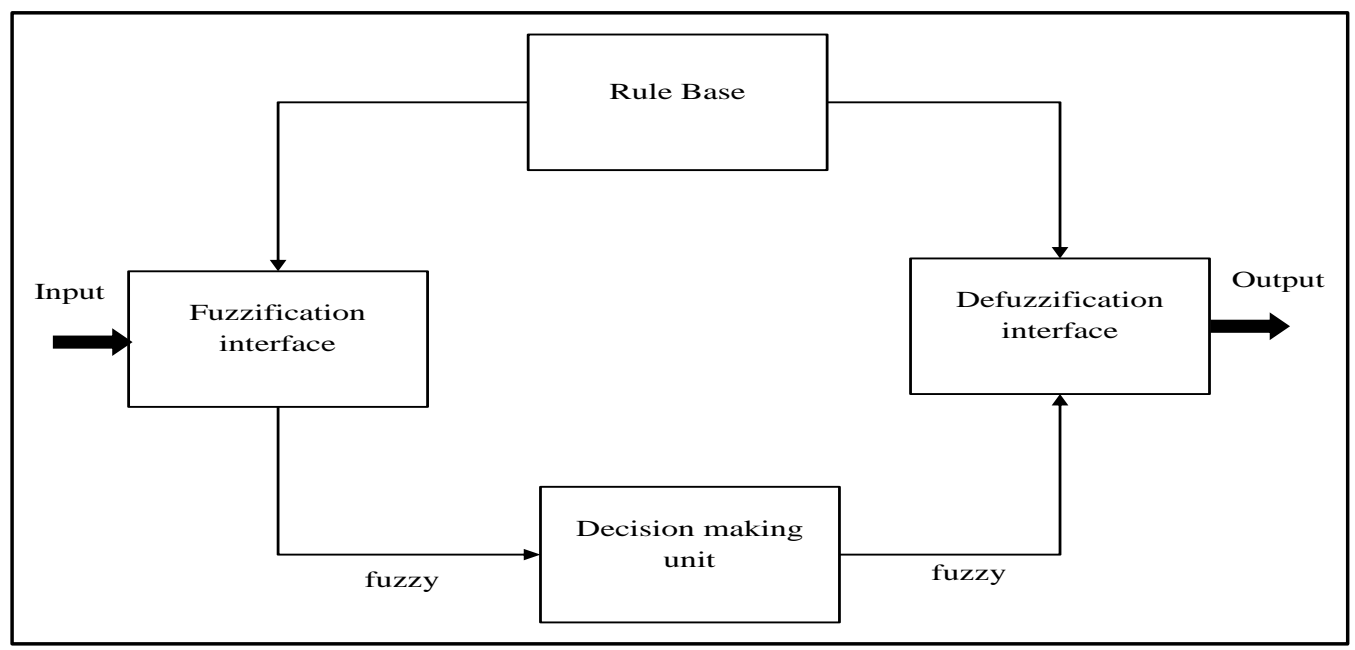

Figure 1.Fuzzification Process

According to the fuzzification process, fuzzy matrix is generated using questionnaires' investigation consistency test. From the generated value cluster head has been selected continuously by comparing the above described parameters. In the cluster creation process, the clusters are basically contained several ordinary and single $\mathrm{CH}$ nodes. A cluster heads typically process as local coordinator for processing clusters and it performs intra-cluster transmission process effective manner. Even though CEPSO with FMPDM method successfully eliminate the load and bandwidth while transmitting data, it must be enhanced by using Honey Bee Optimization With Genetic Algorithm which is explained as follows.

\subsection{Honey Bee Optimization with Genetic Algorithm for Avoid Load and Bandwidth Consumption in WSN}

In this research work, honey bee optimized with genetic algorithm based cluster head selection process is introduced for eliminating the bandwidth consumption and load because that leads to improve the network life time. First searching space is initiated for selecting cluster head. After identifying the searching space, each and every node fitness value is estimated as follows.

Fitness function $=\Sigma_{\mathrm{i}}\left(\mathbf{f}_{\mathrm{i}} \mathbf{X} \mathbf{w i}\right) \forall \mathbf{f}_{\mathrm{i}} \in\left(\mathbf{R}_{\mathrm{fnd}}, \mathbf{R}_{\text {Ind }},-\mathrm{C}\right)$ 
In eqn (16), "Cluster distance C (It is sum of distance from member nodes to Cluster Head and Cluster Head to Base Station), Round which initial node $\operatorname{dies}\left(\mathrm{R}_{\text {fnd }}\right)$,round which final node dies $\left(\mathrm{R}_{\operatorname{lnd}}\right)$ ". Based on the initial fitness value, genetic operators like crossover, mutation and selection process are applied to each node for getting new chromosomes. The generated new chromosomes are interchangd and generate new offspring which is done by crossover operator. Next process is known as mutation, a bit which is defined as ' 0 ' is becomes ' 1 ' similarly ' 1 ' become ' 0 '. The mutation process is employed at the time of finding probability of mutation. This mutation process leads to change normal nodes into cluster nodes and cluster nodes into normal nodes. At this time other nearest cluster nodes are joining into the cluster with appropriate $\mathrm{CH}$ and in case the ordinary node becomes $\mathrm{VH}$, the nodes near then joins the nearest cluster. This process is repeated until to generate the new offspring's by using nodes available in searching space. This searching process is optimized by HoneyBee Optimization (HBO) which has three various collections such as onlooker, employed, and scout bees. These bees are helps to detect the optimal cluster head depending on the food source position fitness value which is estimated as follows.

$$
\mathrm{fit}_{\mathrm{i}}=\frac{1}{1+\mathrm{f}_{\mathrm{i}}}
$$

Where $\mathrm{f}$ is defined as the cost function of giving WSN clustering head creating the problem. Here, the onlooker bee selection of the source of the food depends on the probability value which is connected with that available source of food is computed thru using the following eqn.

$$
P_{i}=\frac{f_{i t_{i}}}{\sum_{n=1}^{S N} f_{i t_{n}}}
$$

Where, SN is defined as the food sources number that is connected with employed bee's available number. At the time of above process, the cluster head and neighboring nodes must satisfy different parameters such as Battery power $(\mathrm{Pv})$, Degree-Difference $(\Delta \mathrm{v})$ and Degree of mobility $(\mathrm{Mv})$. If the selected nodes satisfy these parameter conditions, then it has been selected as the cluster head which helps to determine data transmission nodes by eliminating load and bandwidth. Then the efficiency of power efficient clustering-based data transmission in WSN has been examined using the following experimental results.

\section{Results and Discussion of Experiment}

This part evaluates the efficiency of the proposed optimized algorithm-based cluster head selection methodologies for eliminating the high load, high bandwidth utilization, high energy utilization and network lifetime problem. The excellence of the optimized cluster head selection method is investigated using network lifetime metrics, average power consuming, and ratio of packet delivery. Along with the performance metrics NS2 simulation.

Table 1: Simulation Parameter

\begin{tabular}{|c|c|}
\hline Simulation Parameters & Values \\
\hline Weighting parameters $\left(\alpha_{1}, \alpha_{2}, \alpha_{3}\right)$ & $\mathbf{0 . 2 5 , 0 . 4 5 , 0 . 2}$ \\
\hline Number of particles and ants & 20,20 \\
\hline Simulation period & $1000 \mathrm{~s}$ \\
\hline Number of nodes & 200 \\
\hline Routing protocol & DSR \\
\hline Number of iterations & 260 \\
\hline Initial sensor energy & 150 joules \\
\hline
\end{tabular}




\begin{tabular}{|c|c|}
\hline Sleep power & $\mathbf{0 . 0 0 2}$ watts \\
\hline Transmission power & 12 watts \\
\hline Transmission range & 125 meters \\
\hline Sink node location & $500 \times 500$ \\
\hline Simulation Area & $1000 \times 1000$ sqmeters \\
\hline Reception power & 11 watts \\
\hline
\end{tabular}

According to the simulation parameter, the excellence of the optimized cluster head selection system is evaluated using above described parameter. So, the efficiency of the system is compared with the perspective methods.

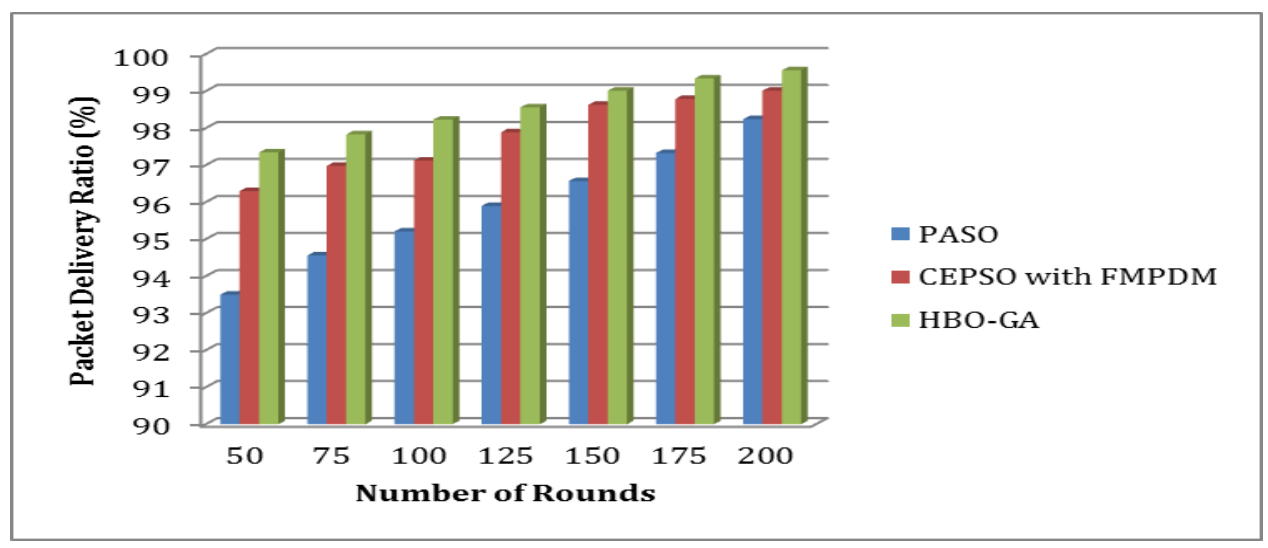

Figure 2: Packet Delivery Ratio

The above figure 2 indicates that the packet delivery ratio of various proposed optimized cluster head selection methods. As shown in figure 2, three proposed methods such as Particle with Ant Swarm Optimization (PASO) technique, Co-Evolutionary Particle Swarm Optimization (CEPSO) algorithm and Honey Bee Optimization with Genetic Algorithm (HBO-GA) transmitted the data effectively from source node to destination node thru utilizing less power that leads to improvise the life-time of network. Even though those three methods high packet delivery ratio, comparing with the methods of PASO and CEPSO with FMPDM method, the method HBO-GA has high packet delivery ratio. In addition, the efficiency of the network is further evaluated with the energy utilization parameter which is shown in the figure 3.

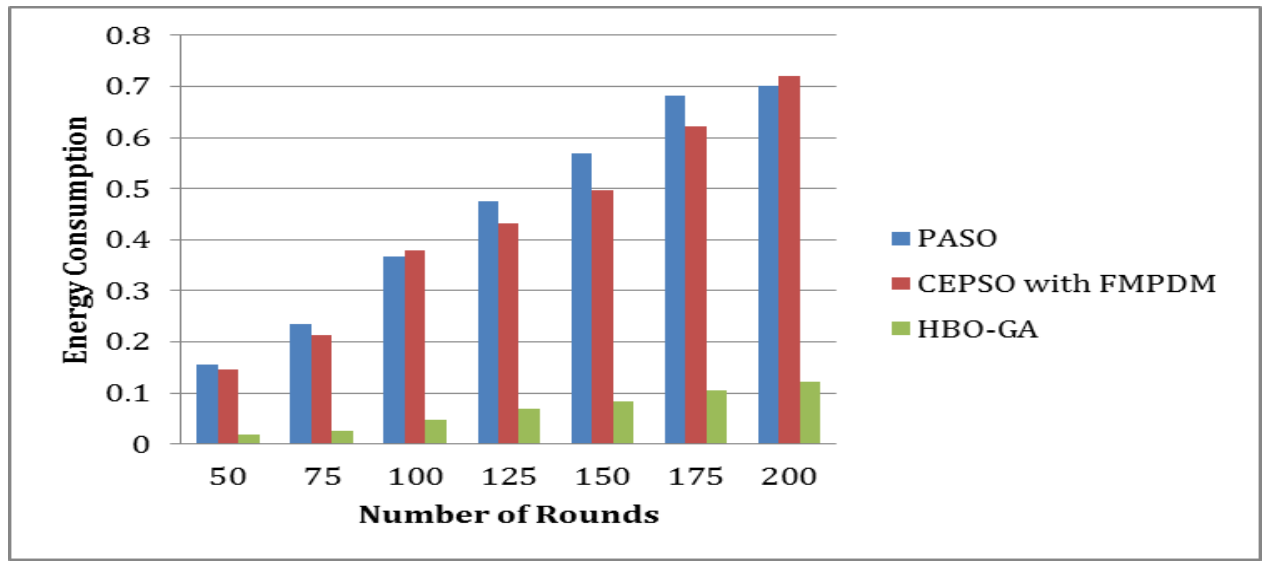

Figure 3 Energy Consumption 
Figure 3 shows Energy consumption analysis of Particle with Ant Swarm Optimization (PASO) technique, CoEvolutionary Particle Swarm Optimization (CEPSO) algorithm and Honey Bee Optimization with Genetic Algorithm (HBO-GA). From the analysis, Honey Bee Optimization with Genetic Algorithm (HBO-GA) based cluster head selection method consumes minimum energy while transmitting data compared with two proposed Particle with Ant Swarm Optimization (PASO) technique, Co-Evolutionary Particle Swarm Optimization (CEPSO). Thus the proposed system effectively utilizes the energy while detecting the optimized route as well as packet transmission. Even though the proposed system consumes minimum energy it must have high network lifetime. Then the proposed system obtain high lifetime of network is explained in the figure 4.

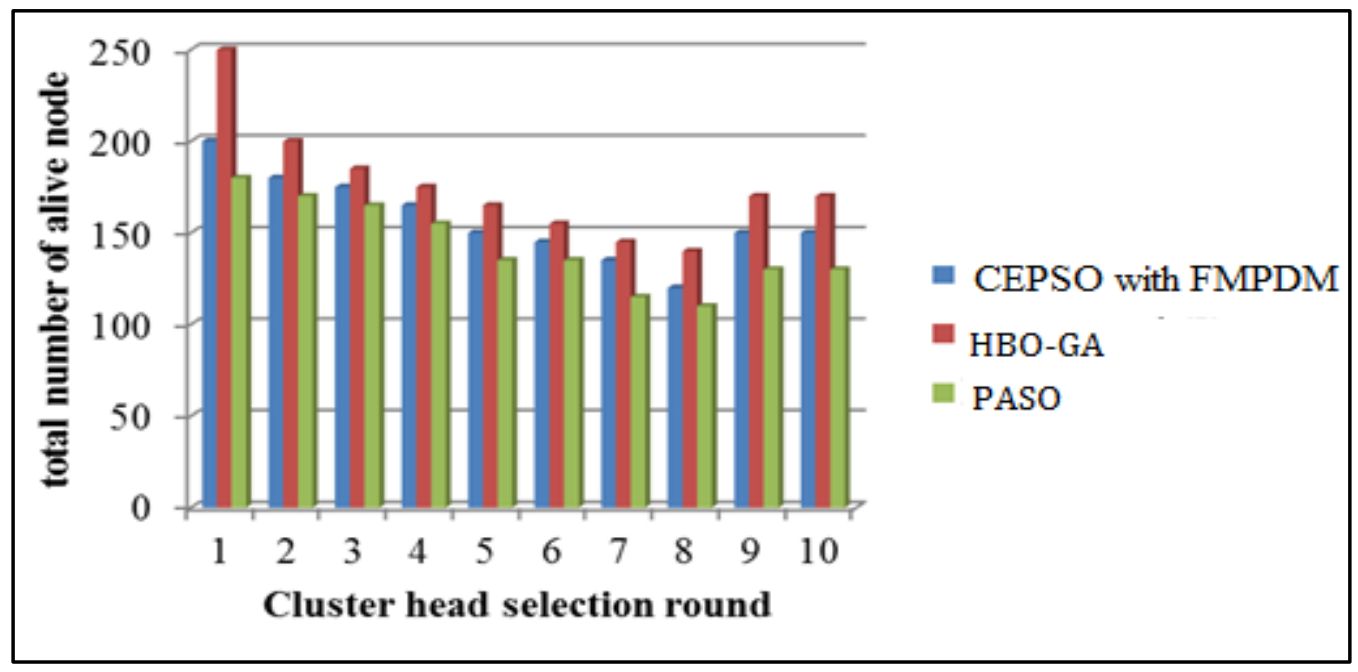

Figure 4: Network Lifetime

The above figure 4 depicted that the network lifetime of the various proposed cluster head selection methods such as Particle with Ant Swarm Optimization (PASO) technique, Co-Evolutionary Particle Swarm Optimization (CEPSO) algorithm and Honey Bee Optimization with Genetic Algorithm (HBO-GA). All the three methods ensure maximum network lifetime which means that the information was successfully transmitted the from source to destination by reducing the network power utilization with high packet delivery ratio. Thus, the above discussions clearly show that the proposed optimized cluster head selection method successfully transmit the data from source to destination by eliminating high utilization of energy, bandwidth and load.

\section{CONCLUSION}

In this research, lifetime and energy of network node has been analyzed in WSN using the Particle with Ant Swarm Optimization (PASO) technique, Co-Evolutionary Particle Swarm Optimization (CEPSO) algorithm and Honey Bee Optimization with Genetic Algorithm (HBO-GA).Initially search space is identified and each node is evaluated with the help of position, velocity, distance values which is examined regarding using fitness value. The calculated fitness value of each node was compared with different parameters such as power, energy, degree-Difference $(\Delta v)$ and Degree of mobility (Mv) values. According to the above parameters, the cluster head is selected; it helps to detect the members during data transition from source to destination process. The system efficiency was evaluated using the results of the experiment in terms of the lifetime of the network, energy consumption, and ratio of packet delivery. Thus, the proposed optimized cluster head selection methodology consumes minimum energy, high maximum network lifetime when compared to other clustering methods.

\section{Reference}

1. Yong Lv, "An Energy Efficient Routing Based on Swarm Intelligence for Wireless Sensor Networks",Journal of Software, Vol. 9, No. 10, 2014.

2. Chaganti B N Lakshmi, S.K.MohanRao, "Enhancing Network Lifetime of a Wireless Sensor Network Using Swarm Intelligence", International Journal of Applied Engineering Research,Vol. 11, No.1, 2016.

3. PoojaNagchoudhury, KavitaChoudhary, "Classification of Swarm Intelligence based Clustering Methods",International Journal of Computer Applications, Vol.91, No.6, PP.28-33, 2014. 
4. Hou, J., Fan, X., Wang, W., Jie, J., \& Wang, Y. "Clustering strategy of Wireless Sensor Networks based on improved Discrete Particle Swarm Optimization", Sixth International Conference on Natural Computation (ICNC), IEEE, Vol. 7, PP. 3866-3870, 2010.

5. Buddha Singh,DayaKrishanLobiyal, "Human-centric Computing and Information Sciences (HCIS)", Vol.2, 2012.

6. Feng, X.; Gao, Z.; Yang, M.; Xiong, "S. Fuzzy distance measuring based on RSSI in wireless sensor network", In Proceedings of the 3rd International Conference on Intelligent System and Knowledge Engineering, Xiamen, China, 17-19, PP. 395-400, 2008.

7. Gholami, M.; Cai, N.; Brennan, R.W,“An artificial neural network approach to the problem of wireless sensors network localization”, Robot. Comput.Integr.Manuf. Vol.29, PP.96-109, 2013.

8. G.Wang, H. Zhu, H. Dai, L. Wu and B. Xiong, "The clustering algorithm of wireless sensor networks based on multi-hop between clusters", Computer Science and Information Engineering, Vol. 3, PP. 66926696, 2008.

9. Puneet Azad, and Vidushi Sharma, "Cluster Head Selection in Wireless Sensor Networks under Fuzzy Environment", Sensor Networks, vol. 2013, article id. 909086, 2013

10. Mohammed A. Mohammed, Ahmed I. Saleh, Mohammed F. Al Rahmawy, "An efficient caching technique for wireless sensor network based on Clustering and data aggregation" International Journal of Scientific \& Engineering Research, Volume 6, Issue 12, December-2015

11. Buddha Singh, DayaKrishanLobiyal, "A novel energy-aware cluster head selection based on particle swarm optimization for wireless sensor networks", Human-centric Computing and Information Sciences (HCIS), vol.2, 2012

12. VarshaGupta ,Shashi Kumar Sharma, "Cluster Head Selection Using Modified ACO", International Conference on Soft Computing for Problem Solving, vol. 335, pp. 11-20, 2014

13. MadhusudhananBaskaran and ChitraSadagopan, "Synchronous Firefly Algorithm for Cluster Head Selection in WSN", The Scientific World Journal, vol. 2015, article id. 780879, pp. 1-7, 2015

14. LustianaPratiwi, Yun-HuoyChoo, AzahKamilahMuda, Noor AzilahMuda, "Improving Ant Swarm Optimization with Embedded Vaccination for Optimum ReductsGeneration", IEEE International Conference on Hybrid Intelligent Systems (HIS), Melacca, pp. 448 - 454, 2011

15. Abbas Karimi, S. M. Abedini, FaranehZarafshan, S.A.R Al-Haddad, "Cluster Head Selection Using Fuzzy Logic and Chaotic Based Genetic Algorithm in Wireless Sensor Network", Journal of Basic and Applied Scientific Research, vol. 4, no. 3, pp. 9694-703, 2013

16. A. Rajagopal, S. Somasundaram, B. Sowmya, T. Suguna, "Soft Computing Based Cluster Head Selection in Wireless Sensor Network Using Bacterial Foraging Optimization Algorithm", International Journal of Electrical, Computer, Energetic, Electronic and Communication Engineering, vol. 9, no. 3, 2015

17. Elhoseny, M., Tharwat, A., Farouk, A., \& Hassanien, A. E. (2017). K-coverage model based on genetic algorithm to extend WSN lifetime. IEEE sensors letters, 1(4), 1-4.

18. Baroudi, U., Bin-Yahya, M., Alshammari, M., \& Yaqoub, U. (2018). Ticket-based QoS routing optimization using genetic algorithm for WSN applications in smart grid. Journal of Ambient Intelligence and Humanized Computing, 1-14.

19. Hussein, M. K., \& Mohammed, M. A. (2018, January). Efficient and accuracy of retrieval files in Elearning system based on click method. In 2018 1st International Scientific Conference of Engineering Sciences-3rd Scientific Conference of Engineering Science (ISCES) (pp. 34-38). IEEE.

20. Hussein, M. K., \& Ali, A. A. (2018). Optimized Quadrant Directional Minimum Energy Consumption Clustering Based Trust Authenticated Routing Protocol for WSN. Journal of Advanced Research in Dynamical \& Control Systems. Vol 1010 Special Issue (pp. 2581-2589).

21. Khalaf, O. I., \& Sabbar, B. M. (2019). An overview on wireless sensor networks and finding optimal location of nodes. Periodicals of Engineering and Natural Sciences, 7(3), 1096-1101.

22. Khalaf, O. I., \& Abdulsahib, G. M. (2019). Frequency estimation by the method of minimum mean squared error and P-value distributed in the wireless sensor network . Journal of Information Science and Engineering , 35(5), 1099-1112

23. Khalaf, O.I., Abdulsahib, G.M., Kasmaei, H.D., Ogudo, K.A. (2020).A new algorithm on application of blockchain technology in live stream video transmissions and telecommunications. International Journal of e-Collaboration, 16(1).

24. Khalaf, O. I., et al,(2020). Optimization of wireless sensor network coverage using the Bee Algorithm. Journal of Information Science and Engineering , 36(2), 377-386 
25. Khalaf, O.I., Abdulsahib, G. M. and Sadik, M., 2018. A Modified Algorithm for Improving Lifetime WSN. Journal of Engineering and Applied Sciences, 13: 9277-9282. 